\title{
LEVANTAMENTO DE DADOS ACERCA DO USO RACIONAL DE MEDICAMENTOS PELOS IDOSOS QUE MORAM SOZINHOS NA CIDADE DE FRAIBURGO - SANTA
}

\author{
CATARINA
}

\section{DATA SURVEY ON THE RATIONAL USE OF MEDICINES BY ELDERLY PEOPLE WHO}

\author{
LIVE ALONE IN FRAIBURGO CITY - SANTA CATARINA
}

Andréa Cristina Dallazem Schweitzer ${ }^{1}$
Bibiana Paula Drambrós ${ }^{2}$
André Leonardo Correa ${ }^{3}$
Mônica Frighetto

Recebido em: 10 jul. 2017

Aceito em: 05 maio 2018

\begin{abstract}
RESUMO: Os idosos representam o grupo etário com maior índice de utilização de fármacos na sociedade. $O$ envelhecimento, sendo um processo biológico, acarreta o desenvolvimento de muitas doenças devido à suscetibilidade natural do organismo. Sendo assim, a terapia medicamentosa deve ser aplicada com cautela no idoso, devido alteração da metabolização dos fármacos comparada à de adultos jovens. O objetivo do estudo foi identificar a forma, frequência e controle com que idosos que vivem sozinhos, no bairro São Miguel, no município de Fraiburgo, Santa Catarina, fazem uso de seus medicamentos. Para a coleta de dados, adotou-se a entrevista através da aplicação de um questionário contendo perguntas fechadas, e que foi aplicado para os 50 idosos nos meses de outubro e novembro de 2016. Todos os indivíduos da amostra faziam o uso de algum medicamento continuamente, incluindo medicamentos para o tratamento da hipertensão arterial sistêmica, hipercolesterolemia, insuficiência renal, tromboembolia e também medicamentos controlados para transtorno da ansiedade e/ou transtorno depressivo maior. Dos entrevistados, $60 \%$ (30) eram do gênero feminino, somente 4\% (2) finalizaram o ensino médio. Em relação a automedicação $80 \%$ declararam que a fazem e $62 \%$ afirmaram já ter tomado algum medicamento por indicação de uma pessoa não especializada. Todos os entrevistados tomam entre 2-12 medicamentos diariamente. Constatou-se padrão elevado de uso de medicamentos entre os idosos que moram sozinhos, resultado preocupante levando em consideração o baixo grau de escolaridade, de entendimento e os problemas de interação medicamentosa, reações adversas que esses medicamentos podem causar quando tomados de maneira irracional.
\end{abstract}

Palavras-chave: Idosos. Automedicação. Uso Racional de Medicamentos.

ABSTRACT: The elderly represents the age group with the highest rate of drug use in society. Aging, being a biological process, causes the development of many diseases due to the natural susceptibility of the organism. Therefore, drug therapy should be applied cautiously in the elderly, due to altered drug metabolism compared to young adults. The objective of the study was to identify the form, frequency and control with

\footnotetext{
1 Universidade do Oeste de Santa Catarina- UNOESC -VIDEIRA.

2 Universidade do Oeste de Santa Catarina- UNOESC -VIDEIRA.

${ }^{3}$ Universidade do Oeste de Santa Catarina- UNOESC -VIDEIRA.

${ }^{4}$ Universidade do Oeste de Santa Catarina- UNOESC -VIDEIRA.
} 
which elderly people living alone in the São Miguel neighborhood in the city of Fraiburgo, Santa Catarina, use their medications. For the data collection, the interview was adopted through the application of a questionnaire containing closed questions, which was applied to the 50 elderly people in the months of October and November of 2016. All individuals in the sample used some medication continuously, including medicines for the treatment of systemic arterial hypertension, hypercholesterolemia, renal failure, thromboembolism and also controlled medications for anxiety disorder and / or major depressive disorder. Of the interviewees, $60 \%$ (30) were female, only $4 \%$ (2) finished high school. Regarding self-medication, $80 \%$ said they did it and $62 \%$ said they had already taken some medicine for the indication of a non-specialist. All respondents take between 2-12 medications daily. It was found a high standard of medication use among the elderly living alone, a worrying result considering the low level of schooling, understanding and problems of drug interaction, adverse reactions that these drugs can cause when taken in an irrational way.

Keywords: Elderly. Self-medication. Rational Use of Medications.

\section{INTRODUÇÃO}

Segundo dados do Instituto Brasileiro de Geografia e Estatística (IBGE, 2016), a população idosa brasileira cresceu quase oito vezes mais em relação aos jovens e quase duas vezes mais que a população total nos últimos 30 anos. Portanto, a estimativa é de que, depois dos já marcados $6,3 \%$ da população total com mais de 60 anos em 1980, as projeções para população idosa extrapolem os 14\%, no ano de 2025. Com estes valores absolutos, o Brasil colocar-se-á como a sexta sociedade com mais idosos do mundo (BEZERRA, 2014).

Acompanhando a evolução geral da idade da população absoluta brasileira, segundo o IBGE, em 2015, o Estado que apresentou a maior expectativa de vida ao nascer foi Santa Catarina (78,7 anos). Isso demonstrou maior expectativa de vida para os homens (75,4 anos) e para as mulheres (82,1 anos) no período, o que sinaliza certa urgência para um aumento na manutenção e assistência a essa faixa etária, que utiliza medicamentos e que demonstra claro crescimento contemporâneo (IBGE, 2016).

Portanto, com o avanço da população idosa no Brasil, sobretudo em Santa Catarina, aumentam-se os desafios aos serviços e aos profissionais da saúde. Como consequência deste avanço geral na média de idade, diversas patologias são encontradas com variadas sintomatologias e, ademais, a ocorrência e prevalência de doenças crônicas degenerativas, as quais normalmente dependem de terapêuticas medicamentosas prolongadas ou contínuas que necessitam de cuidados especiais e determinações específicas em sua manutenção (MOREIRA et al. 2013).

Refletindo o aumento da população de idosos, no Brasil, nota-se um aumento significativo no consumo de medicamentos por esta população, o que leva, dentre outros problemas, o uso incorreto ou desnecessário, resultando em consequências sérias como efeitos colaterais indesejados, reações alérgicas, intoxicações e outros, por parte dos medicados (GALATO et al. 2008). 
Para as pessoas idosas, os riscos envolvidos no consumo de medicamentos são maiores, se comparados às demais camadas da população. No processo do envelhecimento acontecem muitas alterações em nível fisiológico, o que afeta 0 metabolismo das substâncias no organismo, aumentando assim o risco de interação medicamentosa quando muitos medicamentos são administrados juntamente (LUZ, LIMA, MONTEIRO, 2013). Esse fenômeno é observado porque as alterações farmacocinéticas e farmacodinâmicas são afloradas, próprias do processo de envelhecimento, e que tornam esse contingente populacional mais vulnerável às interações entre medicamentos, efeitos colaterais e reações medicamentosas adversas (GOLLARTE et al. 2012). Aconselhamentos permitem um melhor relacionamento entre os profissionais de saúde e 0 paciente, tornando o tratamento mais eficaz e capacita o idoso a lidar com possíveis efeitos colaterais e interações medicamentosas, contribuindo para a adesão do tratamento (ANDRADE, SILVA, FREITAS, 2009).

O maior aconselhamento e o possível acompanhamento de profissionais de saúde diminuiriam os efeitos controversos da automedicação, já que os idosos são os que mais se queixam de problemas relacionados à saúde, e que recorrem com maior facilidade aos medicamentos que tem em casa como alívio dos seus problemas (LUZ, LIMA, MONTEIRO, 2013).

Assim, os medicamentos representam um dos itens mais importantes da atenção à saúde do idoso. As causas de adoecimento e morte neste grupo específico são de etiologia multifatorial e funcional. Associa-se a isso a imensa variedade e disponibilidade de especialidades farmacêuticas e associações de fármacos sem racionalidade terapêutica que justifique a sua comercialização no vasto mercado farmacêutico (FLORES, 2008). Baseando-se neste ponto, percebe-se que as pessoas idosas necessitam de mais informações, porque ao ter tais respostas, é possível evitar muitos eventos nocivos, da qual essas pessoas estão sujeitas. Somadas com uma maior facilidade na aquisição de medicamentos de venda livre, abre-se uma maior possibilidade de o enfermo possuir um estoque variado dos mesmos em casa (LUZ, LIMA, MONTEIRO, 2013).

Desta forma, esse estoque acarreta que as pessoas usem indiscriminadamente tais medicamentos quando os têm em casa, sem conhecer os verdadeiros efeitos do que estão administrando por conta própria. Outro determinante para o agravamento do problema da automedicação caracteriza-se por um atendimento fragmentado ao idoso que não considera, na maioria das vezes, suas especificidades (MANSO, BIFFI, GERARDI, 2015).

Essas deficiências técnicas, somadas a problemas de conhecimento teórico e informação por parte da população idosa, acarreta uma possível predisposição ao uso irracional de medicamentos.

Diante disso, é impossível ignorar a importância de se adotar uma abordagem para as questões ligadas ao uso racional de medicamentos pelos idosos que moram sozinhos. A relevância do tema, a escassez de estudos e o interesse em promover possíveis ajustes para a melhoria da qualidade de vida do idoso, foram os motivos que levaram a autora ao presente desafio que teve como principal objetivo averiguar se o uso de medicamentos é 
feito de uma forma racional na população idosa.

\section{METODOLOGIA}

Trata-se de uma pesquisa quantitativa e exploratória, a qual foi aprovada pelo Comitê de Ética e Pesquisa com Seres Humanos da Universidade do Oeste de Santa Catarina sob o CAEE: 57538916.0.0000.5367 e número do parecer aprovado: 1647599.

A pesquisa foi realizada no bairro São Miguel, do município de Fraiburgo, Santa Catarina, Brasil, com 50 idosos domiciliados, com idade superior a 60 anos entre os meses de outubro e novembro de 2016. Para a coleta de dados, adotou-se a entrevista através da aplicação de um questionário contendo perguntas fechadas. Estas foram apresentadas exclusivamente para os idosos que moravam sozinhos e que recebiam visitas mensais das agentes de saúde do município. Todos os participantes foram orientados sobre o estudo e assinaram o termo de consentimento livre e esclarecido (TCLE), no qual estava delineado o teor da pesquisa.

$O$ instrumento de pesquisa fora elaborado para obtenção de selecionadas características dos pesquisados, que conferem: gênero, escolaridade, se possui auxílio com seus medicamentos, identificação, tipo de uso e se possui medicamentos de uso contínuo, onde os adquirem, hábitos de horário, hábitos de automedicação e se conferem a validade dos medicamentos. Os resultados foram analisados através da frequência de ocorrência e os percentuais das variáveis estudadas.

\section{RESULTADOS E DISCUSSÃO}

O instrumento de pesquisa aplicado para a obtenção de dados no bairro São Miguel, faz referência aos 50 idosos que moram sozinhos. Destes: 60\% (30) pertenciam ao gênero feminino e $40 \%$ (20) e a média de idade dos entrevistados foi de 71,08 anos.

Um importante ponto da análise deste trabalho, já que ao apresentar problemas relacionados à poli farmácia, com o envelhecimento ainda maior da população absoluta, os problemas tenderão a se agravar. Portanto, com o envelhecimento da população, surgemse várias alterações fisiológicas, como a redução de mecanismos homeostáticos e da função hepática, associados à deficiência visual e declínio cognitivo. Degenerações naturais que, dependendo da situação, pode acarretar no aparecimento de reações adversas a medicamentos (MANSO, BIFFI, GIRARDI, 2015).

Em relação ao auxílio com a administração dos medicamentos 33 indivíduos (66\%) afirmaram não receber nenhum tipo de ajuda, como é demonstrado na figura 1.

A problemática associada à administração por conta própria da população pesquisada é o fator-chave, que pode ser ligado ao fato de viverem sós, sem o possível convívio constante de parentes ou cuidadores. Então, notado o envelhecimento progressivo 
da população brasileira, demostram-se necessárias às instaurações de políticas e medidas de saúde direcionadas a essas pessoas e a seus familiares (MOREIRA et al. 2013).

Figura 1 - Distribuição dos idosos que responderam se recebem auxílio na administração de seus medicamentos.

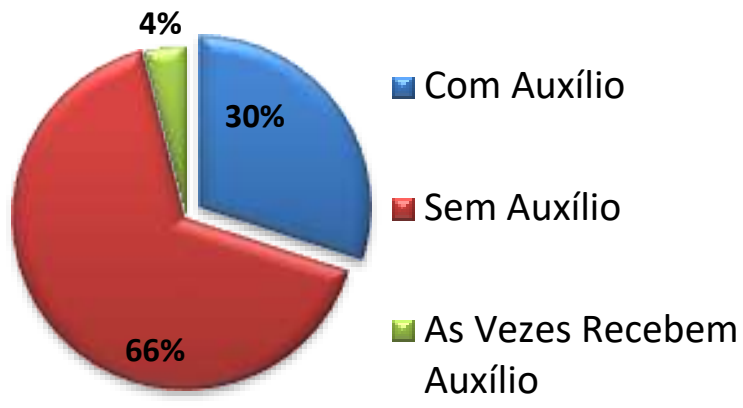

Fonte: Os autores (2017)

Cria-se, portanto, um vínculo entre cuidadores e enfermos, que estarão ligados principalmente na prática da prevenção de doenças e a promoção da saúde, objetivando retardar as modificações decorrentes do envelhecimento da população (MOREIRA et al. 2013).

Em relação ao grau de instrução, apenas 2 (4\%) indivíduos possuem o ensino fundamental completo, enquanto o restante alegou não ter completado a $8^{\circ}$ série (figura 2 ).

Figura 2 - Distribuição do grau de escolaridade dos idosos entrevistados.

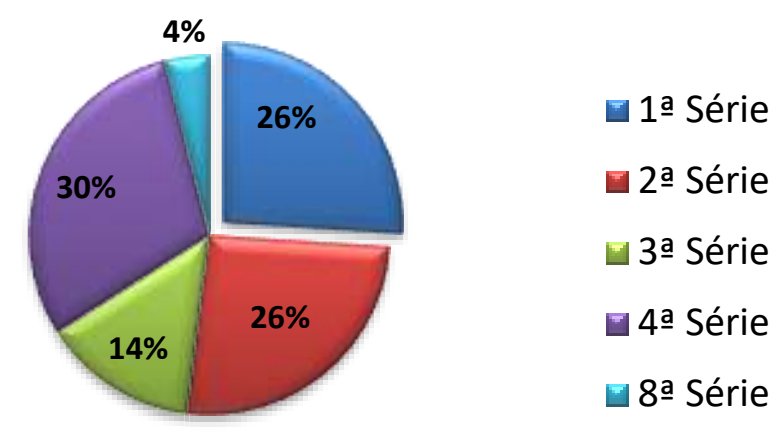

Fonte: Os autores (2017).

Como provável agravante da baixa escolaridade, existe o indício do ingresso prematuro no mercado de trabalho ou na ajuda com os trabalhos manuais no campo, já que a taxa de analfabetismo no campo chega a $23,3 \%$, um número mais de três vezes maior do que a das áreas urbanas, que é de 7,6\%, (IBGE, 2010). Também, segundo o CENSO de 2010, constata-se que a população rural residente de Santa Catarina é de 1.000.485, cifra relativa a mais de $16 \%$ da população total do Estado, superior à média brasileira de $12 \%$.

No que tange a questão da posologia dos medicamentos 21 (42\%) indivíduos da amostra afirmou possuir dificuldade em seu entendimento (figura 3). Logo, sendo a posologia parte crucial da terapêutica, faz-se crer que possivelmente exista uma relação 
entre um baixo teor de ensino demonstrado entre pacientes idosos, sua idade já avançada e um diminuto auxílio na administração diária de medicamentos, explicitado na forma de falta de cuidados específicos (MOREIRA et al. 2013).

Figura 3 - Distribuição da dificuldade no entendimento da posologia dos medicamentos.

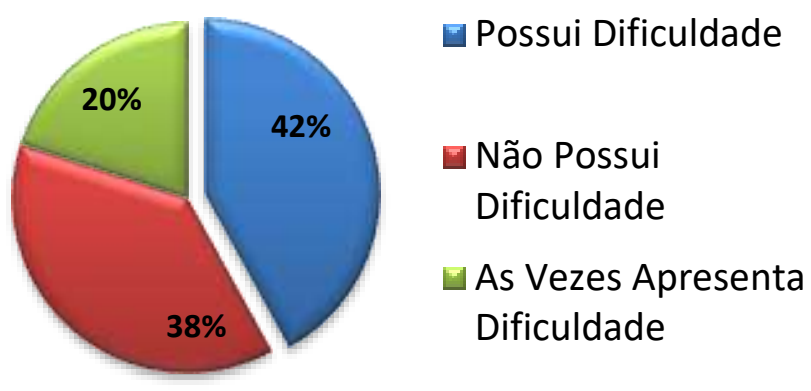

Fonte: Os autores (2017).

Sobre as dificuldades encontradas pela população idosa no entendimento da posologia medicamentosa, e também baseado nos dados do CENSO 2010 (IBGE, 2010) sobre a necessidade de cuidados e cuidadores especializados, Segundo Küchemann (2012), complementa que com o avanço da idade o idoso sofre graduais perdas cognitivas, mudanças comportamentais e emocionais, e que dispõem ativamente do cuidado.

Sobre a regularidade na administração de seus medicamentos, a grande maioria 47 indivíduos (94\%) - alega tomar os medicamentos nos horários corretos, apesar de quando requisitados sobre a utilização de seus medicamentos de uso continuo, 12 (24\%) indivíduos alegaram tomar os medicamentos de forma alternativa à prescrição médica. De forma conjunta, esses dados podem indicar cautela por parte do paciente em manter seus horários padronizados, apesar de $24 \%$ manterem esse padrão de forma diferente à prescrita profissionalmente, como notado na pesquisa (figura 4).

Figura 4 - Índice de idosos que tomam seus medicamentos de uso contínuo diferente da prescrição médica.

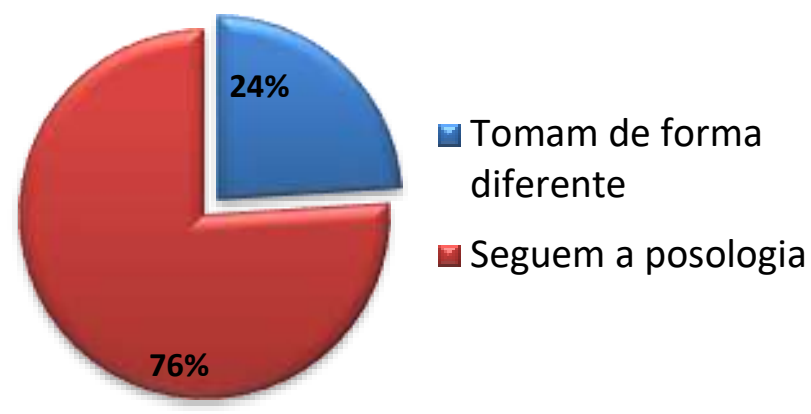

Fonte: Os autores (2017).

Ainda no que tange ao uso de medicamentos nos horários corretos, foi perguntado aos idosos o que faziam com seus remédios quando saiam de casa, 37 (74\%) afirmavam 
levar seus medicamentos junto para que tomem nos horários corretos, 11 (22\%) deixam para tomar todos juntos, quando voltam para casa e os outros 2 (4\%) dividem e tomam alguns antes e outros depois que chegam em casa (figura 5). Segundo Fernandes e Cembranelli (2015), a administração de medicamentos em horários diferentes do prescrito pelo médico, é considerada a principal causa de ineficiência ou alteração dos resultados esperados.

Quando perguntados sobre uso de medicamentos por conta própria, 40 (80\%) indivíduos afirmaram praticar ou já ter praticado a automedicação, e apenas 10 (20\%) indivíduos alegaram não tomar medicamentos por conta e/ou sem ajuda de profissionais.

Infere-se que, como nem sempre o consumo de medicamentos acontece de forma racional, vigora-se o uso inadequado e indiscriminado quanto à automedicação. Segundo Secoli (2010) esta prática é perigosa, porque esconde determinados sintomas das doenças, aumenta a possibilidade de reações adversas, intoxicações e interações medicamentosas.

Figura 5 - Distribuição de como procedem os idosos quanto ao saírem de casa em relação a tomada de medicamentos em seus horários.

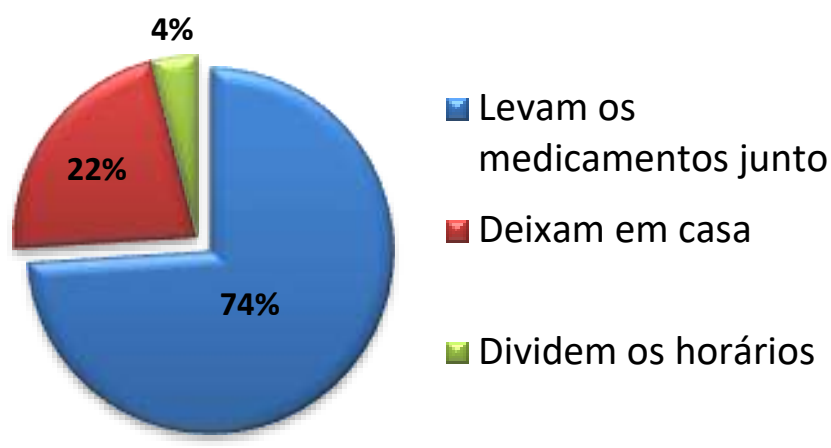

Fonte: Os autores (2017).

Destacado de forma somática ao problema da automedicação, 62\% da amostra pesquisada alegou já ter administrado algum tipo de medicamento por indicação de amigos, vizinhos ou parentes.

Segundo Fernandes e Cembranelli (2015), a prática de automedicação segue como um padrão de prescrições passadas, o que leva a população a adotar critérios próprios para solucionar problemas de saúde de menor gravidade. $O$ mesmo estudo também se dirige a outros fatores envolvidos na automedicação, das quais figuram poder aquisitivo, questões culturais e escolaridade.

$\mathrm{Na}$ pesquisa foi obtida uma média de 5,72 medicamentos diferentes tomados diariamente por indivíduo pesquisado, sendo que, todos os 50 indivíduos faziam uso de pelo menos 2 medicamentos simultâneos continuamente. Com as interações adversas, somadas a administração de variados fármacos simultâneas, o avanço da idade eleva a prevalência de doenças crônicas, destacando, além das doenças cardiovasculares, os cânceres, as doenças respiratórias e as doenças endócrinas, como o diabetes (MANSO, 
2015).

Todos os indivíduos da amostra faziam o uso de algum medicamento continuamente, incluindo medicamentos para o tratamento da hipertensão arterial sistêmica, hipercolesterolemia, insuficiência renal, tromboembolia e também medicamentos controlados para transtorno da ansiedade e/ou transtorno depressivo maior. Com um teto atingindo 12 remédios simultâneos, observado em 2 indivíduos, na tabela 1 está demonstrada a distribuição dos medicamentos continuamente utilizados pelos idosos pesquisados.

Tabela 1 - Distribuição dos medicamentos utilizados continuamente por idosos que moram sozinhos.

\begin{tabular}{|c|c|}
\hline Medicamentos & № / (\%) \\
\hline Hidroclorotiazida 25mg & $27(54 \%)$ \\
\hline Losartana Potássica $50 \mathrm{mg}$ & $25(50 \%)$ \\
\hline Sinvastatina $20 \mathrm{mg}$ & $20(40 \%)$ \\
\hline Omeprazol 20mg & $16(32 \%)$ \\
\hline Ácido Acetil Salicílico 100mg & $9(18 \%)$ \\
\hline Metformina 500mg XR & $7(14 \%)$ \\
\hline Colecalciferol 5mcg + Associações & $7(14 \%)$ \\
\hline Anlodipino $10 \mathrm{mg}$ & $6(12 \%)$ \\
\hline Captopril 25mg & $5(10 \%)$ \\
\hline Enalapril 10mg & $5(10 \%)$ \\
\hline Glibenclamida $5 \mathrm{mg}$ & $5(10 \%)$ \\
\hline Atenolol 25mg & $4(8 \%)$ \\
\hline Metformina $850 \mathrm{mg}$ & $4(8 \%)$ \\
\hline Fumarato de Bormoterol di-hidratado $6 \mathrm{mg}+$ Budesonida $200 \mathrm{mg}$ & $3(6 \%)$ \\
\hline Espirolactona 25mg & $3(6 \%)$ \\
\hline Furosemida $40 \mathrm{mg}$ & $3(6 \%)$ \\
\hline Alendronato de Sódio $70 \mathrm{mg}$ & $3(6 \%)$ \\
\hline Insulina NPH 100UI & $3(6 \%)$ \\
\hline Hemifumarato de Bisoprolol 5mg & $2(4 \%)$ \\
\hline Levotiroxina Sódica $50 \mathrm{mcg}$ & $2(4 \%)$ \\
\hline Levotiroxina Sódica $75 \mathrm{mcg}$ & $2(4 \%)$ \\
\hline Hemifumarato de Bisoprolol $10 \mathrm{mg}$ & $2(4 \%)$ \\
\hline Propranolol 40mg & $2(4 \%)$ \\
\hline Cloridrato de Diltiazen 90mg & $2(4 \%)$ \\
\hline Varfarina Sódica 5mg & $2(4 \%)$ \\
\hline Flunarizina $10 \mathrm{mg}$ & $2(4 \%)$ \\
\hline Aminofilina 100mg & $2(4 \%)$ \\
\hline Hidroclorotiazida 50mg & $1(2 \%)$ \\
\hline Levotiroxina Sódica 88mcg & $1(2 \%)$ \\
\hline Levotiroxina Sódica $125 \mathrm{mcg}$ & $1(2 \%)$ \\
\hline Atorvastatina $10 \mathrm{mg}$ & $1(2 \%)$ \\
\hline Alopurinol 300mg & $1(2 \%)$ \\
\hline Cetotifeno $0,2 \mathrm{mg} / \mathrm{ml}$ & $1(2 \%)$ \\
\hline Cloridrato de Ranitidina 150mg & $1(2 \%)$ \\
\hline Dutasterina $0,5 \mathrm{mg}+$ Tansulosina $04 \mathrm{mg}$ & $1(2 \%)$ \\
\hline
\end{tabular}

№: Número de idosos que utilizavam o medicamento; \% de idosos que utilizavam o medicamento.

Fonte: Os autores (2017).

Segundo Manso (2015) no uso de vários fármacos concomitantemente, aumenta- 
se os riscos de reações adversas, tal qual a probabilidade de gerar problemas com suas interações. Com o uso de dois fármacos ao mesmo tempo a prevalência de efeitos adversos é de $13 \%$, valor que chega a $58 \%$ quando se administram cinco medicamentos, e alcança $82 \%$ quando a farmacoterapia chega a sete ou mais.

Sobre os riscos da polifarmácia e farmacoterapia continuada, Secoli (2010, p.137), aponta que:

(...) é frequente o idoso apresentar de duas a seis receitas médicas e utilizar a automedicação com dois ou mais medicamentos, especialmente para aliviar sintomas como dor e constipação intestinal. Esta situação pode ocasionar eventos adversos, uma vez que o uso simultâneo de seis medicamentos ou mais pode elevar o risco de iterações medicamentosas graves em até 100\% (SECOLI, 2010, p.137).

Como a demonstração da amostra apresentou a utilização de 2 ou mais medicamentos por indivíduo, na tabela 2 está distribuído o percentual dos medicamentos controlados de terapêutica continuada inscritos sob a portaria 344/98 (BRASIL, 1998).

Tabela 2 - Distribuição dos medicamentos controlados sob a portaria 344/98 utilizados continuamente por idosos que moram sozinhos.

\begin{tabular}{ll}
\hline Medicamento & № / (\%) \\
\hline Clonazepan 2,5mg & $5(10 \%)$ \\
Fluoxetina 20mg & $4(8 \%)$ \\
Sertralina 50mg & $3(6 \%)$ \\
Amitriptilina 25mg & $2(4 \%)$ \\
Fenobarbital 100mg & $1(2 \%)$ \\
Alprazolan 1mg & $1(2 \%)$ \\
Citalopran 20mg & $1(2 \%)$ \\
Risperidona 2mg & $1(2 \%)$ \\
Lorazepan 2mg & $1(2 \%)$ \\
Diazepan 5mg & $1(2 \%)$ \\
\hline
\end{tabular}

№: Número de idosos que utilizavam o medicamento; \% de idosos que utilizavam o medicamento. Fonte: Os autores (2017)

Como a polifarmácia está associada ao aumento do risco e da gravidade das Reações Adversas a Medicamentos (RAM's) e das Interações Medicamentosas (IM's), os riscos de causar toxicidade cumulativa, de ocasionar erros de medicação, de reduzir a adesão ao tratamento se elevam (SECOLI, 2010).

Dentre as perguntas realizadas, os entrevistados foram questionados a qual saída optavam quando alguma dúvida surgia a respeito dos medicamentos, ou para quem eles recorriam: majoritariamente 28 (56\%) pediam ajuda a vizinhos ou parentes, enquanto 8 (16\%) alegaram ler e tentar encontrar solução na bula de seus medicamentos, e apenas 14 (28\%) recorriam a postos de saúde ou auxílio de um profissional capacitado. Segundo Gollarte (2012), a atenção farmacêutica para explicar a posologia e sanar todas as dúvidas do paciente na hora da entrega dos medicamentos, é de grande importância para que esses produtos não sejam administrados de maneira incorreta, diminuindo assim todos os problemas com interações e reações adversas. 
Em relação a prática de verificar a validade de seus medicamentos, $30(60 \%)$ afirmaram verificar, enquanto $15(30 \%)$ afirmam não o fazer e $5(10 \%)$ diz ter essa prática de forma esporádica (figura 6).

Figura 6 - Distribuição da aferição da data de validade dos medicamentos afirmadas pelos idosos.

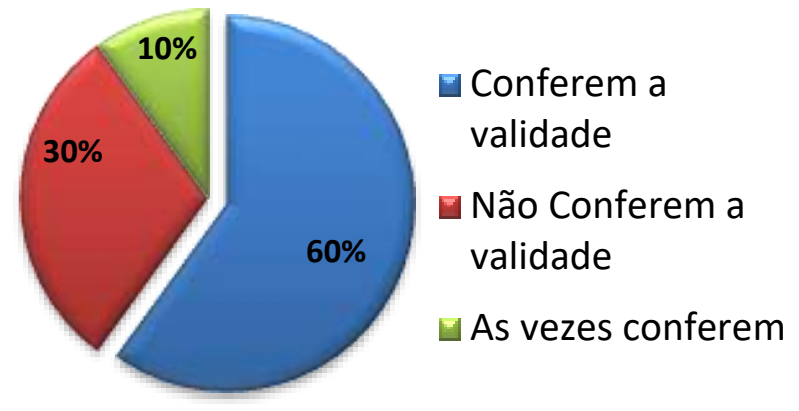

Fonte: Os autores (2017).

Para que os fármacos possam exercer sua ação benéfica desejada e o mínimo de efeitos adversos, cria-se a necessidade de que se mantenham preservadas as condições de estabilidade, que é a propriedade - dentro de limites estabelecidos e sob determinadas condições ambientais - de manter as mesmas características físicas, químicas e farmacológicas, durante seu período de vida útil. Esse espaço de tempo, no qual se assegura sua integridade, é representado como o período de validade do medicamento (SANTIN, VIRTUOSO, OLIVEIRA, 2007). Todos os entrevistados alegaram manter seus medicamentos em suas respectivas embalagens originais, 38 (76\%) dos entrevistados os mantêm na cozinha, fora do acesso a umidade, luz e calor.

Segundo Barcelos (2011), o descarte incorreto de medicamentos vencidos pode acarretar em impactos ambientais extremamente relevantes, afetando diversos ecossistemas. Como mostra a figura 7, somente 11 (22\%) dos idosos levam seus medicamentos vencidos para as farmácias/posto de saúde para que sejam descartados de maneira correta.

Figura 7 - Distribuição dos percentuais dos locais de descarte dos medicamentos vencidos afirmadas pelos idosos.

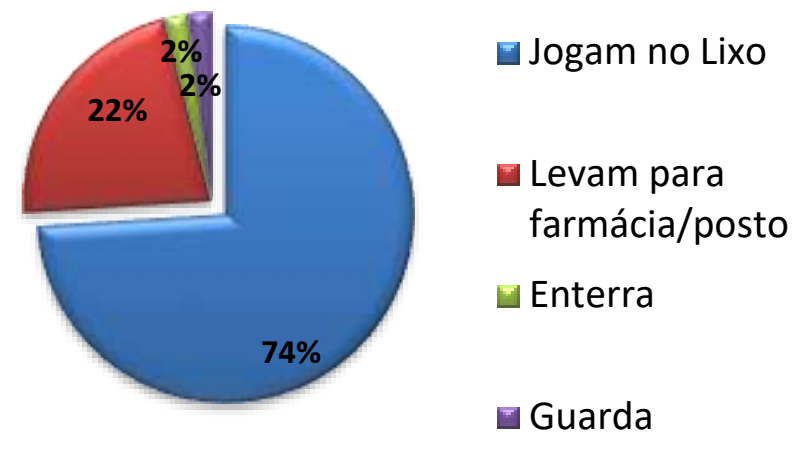

Fonte: Os autores (2017).

Já sobre a prática de instrução de posologias em postos e farmácias, 94\% (47) dos 
entrevistados alegaram que no posto de saúde são instruídos com anotações nas caixas, o que revela outro problema, uma vez que dos entrevistados, 42\% (21) dos entrevistados afirmou apresentar dificuldade com o entendimento das escritas nas caixas e $20 \%$ (10) afirmam, às vezes, não conseguir distinguir o que está escrito.

Ademais, é destacado que a vulnerabilidade dos idosos aos eventos adversos relacionados a medicamentos é bastante alta, o que se deve a complexidade dos problemas clínicos, à necessidade de múltiplos agentes, e às alterações farmacocinéticas e farmacodinâmicas inerentes ao envelhecimento (SECOLI, 2010). Apresentado este panorama, a grande charada dos profissionais da saúde no Brasil, e em especial em Santa Catarina, que está com a população em evidente envelhecimento, é o contribuir na promoção do uso racional dos medicamentos.

A educação dos medicados, especialmente ao grupo que afirma fazer a prática automedicação, pode ser uma opção viável na orientação acerca dos riscos da interrupção, troca, substituição ou inclusão de medicamentos sem conhecimento prévio especializado. Toda essa alternância evitaria a administração simultânea de medicamentos que podem interagir entre si e minimizar os riscos e efeitos adversos apresentados pelos mesmos.

\section{CONCLUSÃo}

A partir dos dados apresentados, observa-se que o uso de medicamentos na população idosa estudada é executado de maneira indiscriminada. Aspectos relevantes foram observados em relação aos idosos e quanto ao uso de medicamentos.

A dificuldade com a posologia pode ser considerada um agravante, já que o nível de instrução da população analisada foi baixo, o que dificulta ainda mais sua autonomia e entendimento relacionado às terapêuticas que estão administrando. Os eventos de consulta a vizinhos e parentes sobre remédios e automedicação tornam-se ainda mais recorrentes, que pode trazer danosas reações à saúde do medicado sem a devida orientação profissional.

Portanto a prática do uso racional de medicamentos na classe idosa precisa ser cada vez mais exposta, tanto para os profissionais da saúde que são responsáveis pelo auxílio, atenção e cuidados com a saúde do paciente, quanto para a própria sociedade, que necessita sempre estar bem informada sobre os cuidados que devem ser empregados para a melhora da qualidade de vida do idoso.

\section{REFERÊNCIAS}

ANDRADE, Marcieni Ataide; SILVA, Marcos Valério Santos; FREITAS, Osvaldo. Assistência Farmacêutica como Estratégia para o Uso racional de Medicamentos em Idosos. Semina: Ciências Biológicas e da Saúde, Paraná, v.25, n.1, p. 55-63, jan. 2009. Disponível em: http://www.uel.br/revistas/uel/index.php/seminabio/article/view/3626. 
Acesso em 10 mai. 2017.

BARCELOS, Mônica Naves, PEREIRA, Ivan de Oliveira, CHAVASCO, Luciana Souza, FREITAS, Daniela Fernanda. Aplicação do método Failure Mode and Effect Analysis (FMEA) na identificação de impactos ambientais causados pelo descarte doméstico de medicamentos Engenharia ambiental. v.8, n.2, p. 62-68, 2011.

BEZERRA, João Carlos Lopes. Facilitação Neuromuscular proprioceptiva em idosos. 2014. 45f Dissertação (Mestrado em Saúde e Sociedade) - Curso de Mestrado em Saúde e Sociedade. UERN, Universidade do Estado do Rio Grande do Norte. Mossoró.

BRASIL. Ministério da Saúde/SNVS. Portaria n³44 de 12 de maio de 1998. Aprova o Regulamento Técnico sobre substâncias e medicamentos sujeitos a controle especial. Diário Oficial da República Federativa do Brasil. Brasília, 31 de dez. de 1998.

FERNANDES, Wendel Simões.; CEMBRANELLI, Julio César. Automedicação e o Uso Irracional De Medicamentos: O Papel Do Profissional Farmacêutico No Combate A Essas Práticas. Revista Univap, São José dos Campos, v. 21, n. 37, jul. 2015.

FLORES, Vanessa Boeira, BENVEGNú,Luís Antônio. Perfil de utilização de medicamentos em idosos da zona urbana de Santa Rosa, Rio Grande do Sul, Brasil. Cad. Saúde Pública, Rio de Janeiro, v.24, n.6, p.1439-1446, jun, 2008.

GALATO, Dayani; ALANO, Graziela Modolon; TRAUTHMAN, Silvana Cristina, VIEIRA, Ana Cristina. A dispensação de medicamentos: uma reflexão sobre o processo para prevenção, identificação e resolução de problemas relacionados à farmacoterapia.

Revista Brasileira de Ciências Farmacêuticas. v.44, n.3, jul/set. 2008.

GOLLARTE Fermin Garcia, JÚLVEZ José Baleriola, LÓPEZ Isabel Ferrero, JENTOFT Alsonso J. Cruz. Inappropriate Drug Prescription at Nursing Home Admission. JAMDA. v. 83, n.13, p. 9-15, 2012.

IBGE, Instituto Brasileiro de Geografia e Estatística. Censo Demográfico 2010: Educação. [online] Disponível em:

<http://www.ibge.gov.br/estadosat/temas.php?sigla=sc\&tema=censodemog2010_educ> Acesso em: 13 de Maio de 2017.

IBGE, Instituto Brasileiro de Geografia e Estatística. Censo Demográfico 2016:

População. [online] Disponível em: <http://www.ibge.gov.br/apps/populacao/projecao/>. Acesso em: 22 de Abril de 2017.

KÜCHEMANN, Berlindes Astrid. Envelhecimento populacional, cuidado e cidadania: velhos dilemas e novos desafios. Revista Sociedade e Estado. v. 27, n.1, p.165-180, jan/abr. 2012.

LUZ, Deolindo João. LIMA, José António Santos, MONTEIRO, Leonel Gomes.

Automedicação no Idoso. 2013. 66.f, Trabalho de Conclusão de Curso (ESCOLA SUPERIOR DE SAÚDE) - CURSO DE ENFERMAGEM, UM, Universidade do Mindelo, Portugal.

MANSO, Maria Elisa Gonzalez; BIFFI, Elaine Cristina Alves, GERARDI, Thiago José. Prescrição inadequada de medicamentos a idosos portadores de doenças crônicas 
em um plano de saúde no município de São Paulo, Brasil. Rev. bras. geriatr. Gerontol, Rio de Janeiro, v.18, n.1, p.151-164. Mar.2015. Disponível em:

<http://www.scielo.br/scielo.php?script=sci_arttext\&pid=S1809-

98232015000100151\&lng=en\&nrm=iso >. Acesso em 20 abr. 2017.

MOREIRA, Ramon Missias, SANTOS, Carla Elane Silva; COUTO, Edvaldo Souza;

TEIXEIRA, Jules Ramon Brito, SOUZA, Riane Missias Moreira Mendes. (2013, março).

Qualidade de vida,Saúde e Política Pública de Idosos no Brasil: uma reflexão teórica.

Revista Kairós Gerontologia. v.16, n.2, p.27-38. 2013.

SANTIN, Patrícia Oliveira Rocha, VIRTUOSO, Suzane, DE OLIVEIRA, Simone Maria Menegatti. Farmácia Domiciliar: Uma Caixa De Surpresas. Visão Acadêmica, Curitiba, v.8, n.2, jul/dez. 2007.

SECOLI, Silvia Regina. Polifarmácia: interações e reações adversas no uso de medicamentos por idosos. Rev. bras. Enferm. Brasília, v. 63, n. 1, p. 136-140, Feb. 2010. Disponível em: http://www.scielo.br/scielo.php?script=sci_arttext\&pid=S0034$71672010000100023 \&$ Ing=en\&nrm=iso $>$. Acesso em 7 mai. 2017. 